\title{
Non-Abelian geometrical control of a qubit in an NV center in diamond
}

\author{
Jing $\mathrm{Lu}^{1}$ and Lan Zhou, ${ }^{1, *}$ \\ ${ }^{1}$ Key Laboratory of Low-Dimensional Quantum Structures and Quantum Control of Ministry of Education, \\ and Department of Physics, Hunan Normal University, Changsha 410081, China
}

\begin{abstract}
We propose an approach for an optical qubit rotation in the negatively charged nitrogen-vacancy $(\mathrm{NV})$ center in diamond. The qubit is encoded in the ground degenerate states at the relatively low temperature limit. The basic idea of the rotation procedure is the non-Abelian geometric phase in an adiabatic passage, which is produced by the nonadiabatic transition between the two degenerate dark states. The feasibility is based on the success of modeling the NV center as an excited-doublet four-level atom.
\end{abstract}

PACS numbers: 42.50.Ex, 61.72.-y, 03.65.Vf

\section{INTRODUCTION}

Phases play a major role in all interference and diffraction phenomena in optics, wave physics and quantum mechanics. The study of phase has generated a large number of practical device [1]: superconducting interference device, interferometers to measure lengths and small rotations, modulators to modulate wavefront, etc. In theoretical physics, phases are involved in the distinction between fermions and bosons, decoherence of a quantum system and Aharonov-Bohm effect. It is well known that a quantum system acquire a Berry phase [2] which evolves adiabatically around a circuit by varying parameters in its Hamiltonian. This phase is a sum of two parts. The first one is related to the instantaneous energy of the system, which is called dynamical phase. The second one is a purely geometric property of the closed path followed by the system in space, which is called geometrical phase. Later, non-Abelian gauge fields arising in the adiabatic development of simple quantum mechanical systems are introduced by Wilczek et al [3]. Nowadays, the Berry phase is not only of interest from a fundamental point of view, for example, the induced gauge field [4, 5] and topological states of matter [6], but also may have important applications in quantum information processing. Holonomic quantum computation [7] is a prototype of research on quantum gates based on Abelian or nonAbelian geometric phases. Its significant attraction lies on that these quantum gates could be inherently robust against some local perturbations. Moreover, interest are paid on the adiabatic evolution of dark eigenstates of the considered system in order to remove any accompanied dynamical phase shift.

On the other hand, the negatively charged nitrogenvacancy $(\mathrm{NV})$ center in diamond has recently emerged as a promising candidate for practical and scalable implementation of quantum information processing due to the following robustness: it is an individually addressable quantum system; its quantum state can be initialized

*Electronic address: zzhoulan@gmail.com and manipulated with optical and microwave fields, and even measured with high fidelity at room temperature 8 12]. NV centers are formed by a substitutional nitrogen atom adjoining to a vacancy in the diamond lattice. The dangling bonds near the vacancy are occupied by six electrons, which includes three dangling bonds on the carbon atoms and two bonding bonds on the nitrogen atom [13]. The nitrogen atom breaks down the $T_{d}$ symmetry and gives rise to the trigonal symmetry [14, 15]. A single NV center have a long-lived spin triplet in its electronic ground state whose levels function as a qubit and are tunable with an applied magnetic field [16]. Currently, the applications rely on the spin in the ground state, for example, few-qubit networks for simple algorithms and quantum memories are created by coherently coupling NV-center spins to nearby electronic [17, 18] and nuclear spins [9, 19]; the spin triplet ground state also acts as a sensitive magnetic probe of the local environment 20-22]. Optical techniques constitute powerful tools in quantum physics and quantum information science 23, 24]. It is known for a considerable time that the NV in its singly-charged state is good single-photon source [25]. Nowadays, its optical Rabi oscillations have been observed [26]. Coherent population trapping using optical laser fields has been found in single NV centers [27]. And the crucial element for solid-state realization of quantum optical networks has been demonstrated by the entanglement between the polarization of a single optical photon and a solid-state qubit associated with the single electronic spin of a NV center in diamond [28].

An important advantage of optical techniques in comparison with microwave manipulation is spatial resolution which can selectively address a single NV defect in diamond as well as individual qubits. Here, we propose an scheme to achieve an qubit rotation by the coherent interaction of light and single NV centers in diamond. The qubit is encoded in the degenerate Zeeman sublevels in the absence of external strain and electric or magnetic fields. Due to the spin-orbit and spin-spin interactions, an excited-double four-level system can be established in the spin-triplet ground state and an orbital-doublet, spintriplet excited state of the NV center, which allow us to apply two pump and two Stokes laser pulses. The rotation of qubit based on the non-Abelian geometric phase 
is realized by tuning adiabatically the Rabi frequencies and phases of the pump and Stokes pulses.

The paper is organized as follows. In Sec. II the energy levels of a NV center is briefly reviewed and the excited-double four-level system is modeled for a NV center with an electronic spin triplet in the ground state and an orbital-doublet, spin-triplet excited state. In Sec. III we present our method for arbitrary qubit rotation. Summaries are made in Sec. IV.

\section{FORMULATION OF AN EXCITED-DOUBLE FOUR-LEVEL SYSTEM}

NV centers consist of a substitutional nitrogen atom and an adjacent vacancy, which are a naturally occurring defect in diamond with highly localized electronic bound states. The bound states of NV centers are multiparticle states of six electrons: five contributed by the nearest-neighbor nitrogen and carbon atoms to the vacancy and an additional captured from the bulk. To find out the electronic states of NV centers, single-electron molecular orbitals are employed, which are built up from linear combinations of the dangling sp3 orbitals of one nitrogen and three carbon atoms around the vacancy. Applying group theoretical arguments to the NV center leads to four molecular orbitals $\left.\left\{u, v, e_{x}, e_{y}\right\} 13-15\right]$. Orbitals $u$ and $v$ are totally symmetric but non-degenerate which transform according to the one-dimensional irreducible representation of $C_{3 v}$ symmetry, and the two degenerate orbitals $\left\{e_{x}, e_{y}\right\}$ transform according to the two-dimensional irreducible representation of $C_{3 v}$ point group. The ordering of the orbitals are obtained by symmetry and charge considerations of the electron-ion interaction, i.e. $u$ orbital has the lowest energy, $v$ is the next lowest orbital and doubly degenerate $e_{x, y}$ orbitals are the highest energy. Filling four orbitals with six electrons according to the Pauli exclusion principle indicates that the ground state consists of four electrons completely occupying the totally symmetric orbitals and two electrons occupying the remaining orbitals. Although the spin-obit wave functions for the ground-state configuration give ${ }^{3} A_{2},{ }^{1} A_{1}$ and ${ }^{1} E$ states due to the antisymmetry property of the total wavefunction for fermionic particles, Hund's rules predict that the ground state is attributed to the spin-triplet ${ }^{3} A_{2}$ state. The excited state configuration can be learned from one electron being promoted from the $v$ orbital to the $e_{x, y}$ orbitals in the single particle picture [12]. The excited-state configuration has an orbital-doublet, spinsinglet ${ }^{1} E$ state and an orbital-doublet, spin-triplet ${ }^{3} E$ state with manifold $\left\{E_{x}, E_{y}, E_{x}^{\prime}, E_{y}^{\prime}, A_{1}, A_{2}\right\}$ according to the irreducible representation of $C_{3 v}$ symmetry. The degeneracy between triplets and singlets in the groundstate and excited-state configurations are lifted by the electron-electron Coulomb interaction. Nowadays, it is known that the optical transition is associated with the ${ }^{3} A_{2}$ ground state and ${ }^{3} E$ excited state. The spin-spin in-

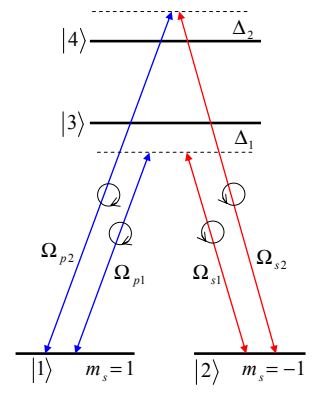

FIG. 1: (Color online) The energy diagram and selection rule of the excited-doublet four-level system associated with the ground and excited states in the negatively charged nitrogenvacancy center.

teraction causes the $2.87 \mathrm{GHz}$ zero field splitting between the magnetic sublevels $m_{S}=0$ and $m_{S}= \pm 1$ states of the ${ }^{3} A_{2}$ ground triplet state. The spin-orbit interaction splits the ${ }^{3} E$ excited state into three doublet degenerate states, noted by $E, E^{\prime}, A_{1}$ and $A_{2}$. And the degeneracy of the $A_{1}$ and $A_{2}$ states is lifted by the spin-spin interaction.

The $\sigma_{+}\left(\sigma_{-}\right)$-polarized optical pulse drives the transition between the ground $m_{s}=-1(+1)$ state with the $A_{1}$ and $A_{2}$ states. Neglecting the $\sigma_{+}\left(\sigma_{-}\right)$-polarized optical coupling between the ground $m_{s}=1(-1)$ state and the $E_{x}$ and $E_{y}$ states and the $x(y)$-polarized optical coupling between the ground $m_{s}=0$ state and the $E_{y}^{\prime}\left(E_{x}^{\prime}\right)$, a excited-doublet four-level system is therefore configured in the absence of external strain and electric or magnetic fields, whose energy diagram and selection rule are schematically illustrated in Fig. 11 The ground states with $m_{s}= \pm 1$ are denoted by $|1\rangle$ and $|2\rangle$, and the excited states are denoted by $|3\rangle$ and $|4\rangle$. The transitions from states $|1\rangle$ with energy $\omega_{1}$ and $|2\rangle$ with energy $\omega_{2}$ to the upper two states with energy $\omega_{3}$ and $\omega_{4}$ are driven by the pump fields and the Stokes laser fields, respectively. The pump field is a $\sigma_{-}$-polarized bichromatic wave with frequencies $\nu_{p 1}$ and $\nu_{p 2}$. And the Stokes field is a $\sigma_{+^{-}}$ polarized bichromatic wave with frequencies $\nu_{s 1}$ and $\nu_{s 2}$. The Hamiltonian for this system is then

$$
\begin{aligned}
H & =\omega_{1}|1\rangle\left\langle 1\left|+\omega_{2}\right| 2\right\rangle\left\langle 2\left|+\omega_{3}\right| 3\right\rangle\left\langle 3\left|+\omega_{4}\right| 4\right\rangle\langle 4| \\
& +\frac{i}{2}\left(\Omega_{p 1} e^{-i \nu_{p 1} t}-\Omega_{p 2} e^{-i \nu_{p 2} t}\right)(|1\rangle\langle 3|-| 1\rangle\langle 4|)+\text { h.c. } \\
& -\frac{i}{2}\left(\Omega_{s 1} e^{-i \nu_{s 1} t}+\Omega_{s 2} e^{-i \nu_{s 2} t}\right)(|2\rangle\langle 3|+| 2\rangle\langle 4|)+\text { h.c. }
\end{aligned}
$$

where $\Omega_{p i}$ and $\Omega_{s i}$ are time-dependent Rabi frequencies where a $\pi$ phase difference has been introduced in the pump field. By assuming that frequencies $\nu_{p i}=\nu_{s i}$, we can define two-photon detunings $\Delta_{1}=\omega_{3}-\omega_{1}-\nu_{p 1}$ and $\Delta_{2}=\nu_{p 2}+\omega_{1}-\omega_{4}$ due to the degeneracy of the ground state. In the rotating frame with respect to

$$
H_{0}=\sum_{i=1}^{2} \omega_{i}|i\rangle\left\langle i\left|+\left(\omega_{3}-\Delta_{1}\right)\right| 3\right\rangle\left\langle 3\left|+\left(\omega_{4}+\Delta_{2}\right)\right| 4\right\rangle\langle 4|,
$$




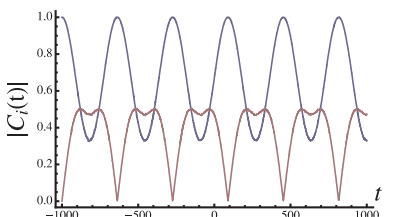

(a)

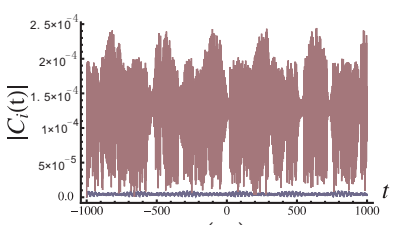

(c)

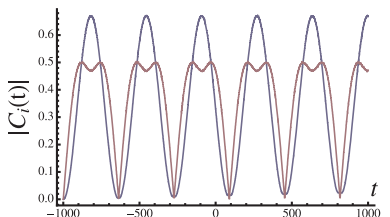

(b)

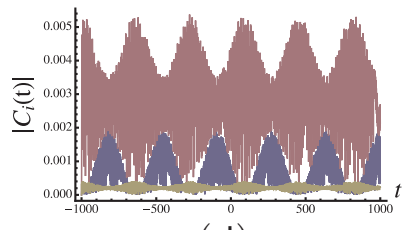

(d)
FIG. 2: (Color online) The norm of probability amplitudes for the ${ }^{3} A_{2}$ ground and ${ }^{3} E$ excited states as function of time when $\mathrm{NV}$ center is initially in the $m_{S}=-1$ state of the ${ }^{3} A_{2}$ ground triplet state, (a) for $m_{S}=-1$ ground (blue) and $A_{2}$ excited (red) states, (b) $m_{S}=1$ ground (blue) and $A_{1}$ excited (red) states. The norm of the amplitude for $m_{S}=0$ ground state is plotted in (c) with blue. The superpositions of states $E_{x}^{\prime}$, $E_{x}, E_{y}$ and $E_{y}^{\prime}$ give rise to other four states whose norms are shown in (c) with red color and (d). All Rabi frequencies are taken as $14 \mathrm{MHz}$. And detunings $\Delta_{1}=\Delta_{2}=10 \mathrm{MHz}$. Other spin-orbit and spin-spin parameters are taken from Ref. [14]. The time axis is in units of ns.

the Hamiltonian in the interaction picture is obtained as

$$
H_{I}=\left[\begin{array}{cccc}
0 & 0 & \frac{i}{2} \Omega_{p 1} & \frac{i}{2} \Omega_{p 2} \\
0 & 0 & -\frac{i}{2} \Omega_{s 1} & -\frac{i}{2} \Omega_{s 2} \\
-\frac{i}{2} \Omega_{p 1}^{*} & \frac{i}{2} \Omega_{s 1}^{*} & \Delta_{1} & 0 \\
-\frac{i}{2} \Omega_{p 2}^{*} & \frac{i}{2} \Omega_{s 2}^{*} & 0 & -\Delta_{2}
\end{array}\right]
$$

by employing the rotating-wave approximation.

To justify that it is valid to neglect optical coupling between the ground $m_{s}=1(-1)$ state and the $E_{x}$ and $E_{y}$ states, as well as the coupling between the ground $m_{s}=0$ state and the $E_{y}^{\prime}\left(E_{x}^{\prime}\right)$, we take the spin-orbit and spinspin parameters from Ref. 14]. Besides, we set all Rabi frequencies $\Omega_{p i}$ and $\Omega_{s i}$ have the same value $14 \mathrm{MHz}$, and detunings $\Delta_{1}=\Delta_{2}=10 \mathrm{MHz}$. Then we numerically solve the time-dependent Schrödinger equation for the spin-triplet ${ }^{3} A_{2}$ state and orbital-doublet, spin-triplet ${ }^{3} E$ state with the system initially in the $m_{S}=-1$ state of the ${ }^{3} A_{2}$ ground triplet state. Figure 2 numerically illustrates the norm of probability amplitudes as a function of time for (a) $m_{S}=-1$ ground (blue) and $A_{2}$ excited (red) states, (b) $m_{S}=1$ ground (blue) and $A_{1}$ excited (red) states. The norm of the amplitude for $m_{S}=0$ ground state is plotted in Fig.(c) with blue. The superpositions of states $E_{x}^{\prime}, E_{x}, E_{y}$ and $E_{y}^{\prime}$ give rise to other four states whose norms are shown in Fig. 2(c) with red and (d). It can be found that the probabilities on the ground $m_{S}= \pm 1$ states and $A_{1}, A_{2}$ excited states are much larger than those of other states. Therefore, we can eliminate the contribution of the other states and model the NV centers as excited-doublet four-level systems.

\section{QUBIT ROTATION}

We now find the eigenstates of Hamiltonian (3). The dark states would be desirable for gate operation in order to remove the dynamic phase shift. Dark states are zero-eigenvalue eigenstates of a considered system. The characteristic equation of this excited-doublet four-level system shows that two eigenvalues of Eq.(31) will be zero if we let frequency detunings $\Delta_{1}=\Delta_{2}=\omega_{0}, \Omega_{p i}=\Omega_{p}$ and $\Omega_{s i}=\Omega_{s}$. And these two corresponding dark states $\left|D_{1}\right\rangle$ and $\left|D_{2}\right\rangle$ are coherent superpositions of the bare states and have the following expression

$$
\begin{aligned}
& \left|D_{1}\right\rangle=\frac{\Omega_{s}^{*}}{\Omega}|1\rangle+\frac{\Omega_{p}^{*}}{\Omega}|2\rangle, \\
& \left|D_{2}\right\rangle=\frac{i \Omega}{\sqrt{2} \Theta}(|3\rangle-|4\rangle)+\sqrt{2} \frac{\omega_{0}}{\Theta}\left|B_{1}\right\rangle,
\end{aligned}
$$

where $\Omega=\sqrt{\left|\Omega_{s}\right|^{2}+\left|\Omega_{p}\right|^{2}}$ and $\Theta=\sqrt{\Omega^{2}+2 \omega_{0}^{2}}$. Here, the bright state

$$
\left|B_{1}\right\rangle=\frac{\Omega_{p}}{\Omega}|1\rangle-\frac{\Omega_{s}}{\Omega}|2\rangle
$$

is orthogonal to the dark state $\left|D_{1}\right\rangle$.

Let us first consider that the applied pump and Stokes fields are in resonance with its corresponding atomic transition, and two-photon resonance is still maintained. In this case, we have $\omega_{0}=0$. The dark state $\left|D_{2}\right\rangle$ is coherent superpositions of the bare states $|3\rangle$ and $|4\rangle$, which leads to $\left\langle D_{i}\left|\partial_{t}\right| D_{j}\right\rangle=0$ for $i \neq j$, i.e. the non-adiabatic transition between the two degenerate dark states vanishes. Then, one can completely transfer the population from $|1\rangle$ to $|2\rangle$ vice versa by the techniques of Stimulated Raman adiabatic passage since the pump and Stokes field are involved in the dark state $\left|D_{1}\right\rangle$.

When the applied fields are off resonance, i.e., $\omega_{0} \neq 0$, there is a non-adiabatic transition between these two dark states, which plays the important role in singlequbit gate operation. Notice that states $|1\rangle$ and $|2\rangle$ span the same eigenspace as the dark state $\left|D_{1}\right\rangle$ and bright state $\left|B_{1}\right\rangle$, and the qubit described by $\alpha|1\rangle+\beta|2\rangle$ does not change with time when $\Omega_{s}=\Omega_{p}=0$, we can project the qubit on the dark state $\left|D_{1}\right\rangle$ and bright state $\left|B_{1}\right\rangle$. The projection requires the Rabi frequencies $\Omega_{p}$ and $\Omega_{s}$ to have essentially the same envelopes

$$
\begin{aligned}
& \Omega_{p}(t)=\Omega(t) \cos \chi e^{i \phi} e^{i \psi(t)}, \\
& \Omega_{s}(t)=\Omega(t) \sin \chi e^{-i \phi} e^{i \psi(t)},
\end{aligned}
$$

Here, $\chi$ and $\phi$ are time-independent angles, which specify the Euler angle of a vector

$$
|\mathbf{D}\rangle=\sin \chi e^{-i \phi}|1\rangle+\cos \chi e^{i \phi}|2\rangle
$$

The first dark state and bright state can be written as

$$
\left|D_{1}\right\rangle=e^{-i \psi(t)}|\mathbf{D}\rangle,\left|B_{1}\right\rangle=e^{i \psi(t)}|-\mathbf{D}\rangle .
$$


We denote the Bloch sphere containing vectors $|\mathbf{D}\rangle$ and $|-\mathbf{D}\rangle$ as BS1. The fixed angles $\chi$ and $\phi$ keep vectors $|\mathbf{D}\rangle$ and $|-\mathbf{D}\rangle$ as constant vectors in BS1 during the time evolution. Hence, any initial state of the qubit can be written as a superposition of $|\mathbf{D}\rangle$ and $|-\mathbf{D}\rangle$. The mutually orthogonal state vectors $|-\mathbf{D}\rangle$ and $|\mathbf{0}\rangle \equiv(|3\rangle-|4\rangle) / \sqrt{2}$ construct the north and south pole of another unit 2sphere denoted as BS2. The second dark state $\left|D_{2}\right\rangle$ is a vector in BS2, whose adiabatic evolution can be written as

$$
\left|D_{2}\right\rangle=i \sin \varphi(t)|\mathbf{0}\rangle+e^{i \psi(t)} \cos \varphi(t)|-\mathbf{D}\rangle,
$$

where $\tan \varphi=\Omega /\left(\sqrt{2} \omega_{0}\right)$. Since the two photon detuning is always on, the adiabatic pulses force the system to remain in dark states. However, vector $\left|D_{2}\right\rangle$ moves slowly with time due to its time-dependent azimuthal and pole angles. When the pump and Stokes pulses are adiabatically turned on, the eigenstate $\left|D_{2}\right\rangle$ evolves smoothly from the initial state $|-\mathbf{D}\rangle$ to a intermediate state which is a superposition state of $|\mathbf{0}\rangle$ and $|-\mathbf{D}\rangle$. The inverse process, i.e. adiabatically turning of the pump and Stokes pulses, brings the system back to its initial state $|-\mathbf{D}\rangle$. When angles $\varphi$ and $\psi$ make a cyclic evolution, the path of the $\left|D_{2}\right\rangle$ vector encloses a patch on the BS2 with a solid angle $\gamma_{c}$, which is the Berry phase. This cyclic evolution gives rise to a phase change on the state $|-\mathbf{D}\rangle$. The unitary operator

$$
U_{C}=e^{i \gamma_{C}}|-\mathbf{D}\rangle\langle-\mathbf{D}|+| \mathbf{D}\rangle\langle\mathbf{D}|
$$

depicts the effect of the cyclic evolution on the vectors in BS1. In the bare basis $|1\rangle$ and $|2\rangle$, the unitary operator

$$
U_{C}=e^{i \gamma_{c} / 2}\left[\cos \frac{\gamma_{c}}{2} \hat{I}+i \sin \frac{\gamma_{c}}{2} \hat{n} \cdot \hat{\sigma}\right]
$$

rotates the qubit encoded in states $|1\rangle$ and $|2\rangle$ about the axe

$$
\hat{n}=(-\sin 2 \chi \cos 2 \phi,-\sin 2 \chi \sin 2 \phi, \cos 2 \chi)
$$

by an angle $\gamma_{c}$ apart from a global phase $\gamma_{c} / 2$, where $\hat{\sigma}=\left(\sigma_{x}, \sigma_{y}, \sigma_{z}\right)$ are the pauli's spin operators. Hence, arbitrary rotation of the qubit can be accomplished by an adiabatic process.

We now design a rotation of the qubit about $x$ with an angle $\pi / 2$ to support the above analysis. The rotation about axe $x$ is obtained by choosing fixed angles $\chi=$ $-\pi / 4$ and $\phi=0$ in Eq.(6). For an adiabatic evolution, $\Omega(t)$ and $\psi(t)$ are required to be smooth functions which vanish at infinity. We take the envelope and phase of Rabi frequencies as follow

$$
\begin{aligned}
\Omega(t) & =\frac{\sqrt{3} \omega_{0} \sin \eta}{\sqrt{2-\frac{3}{2} \sin ^{2} \eta}}, \\
\psi(t) & =\frac{\pi}{2}-\arctan \frac{2 \cos \eta}{\sin \eta},
\end{aligned}
$$

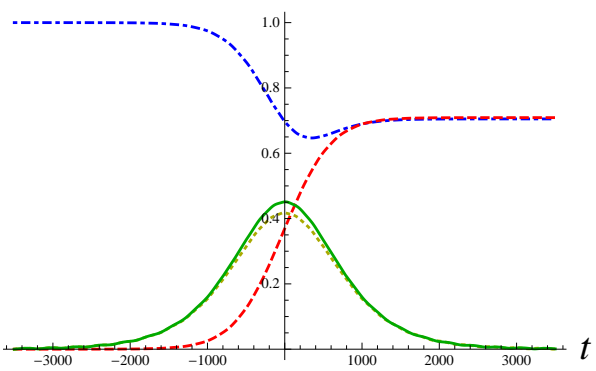

FIG. 3: (Color online) The norm of amplitudes for state $|1\rangle$ (blue dot-dashed line), $|2\rangle$ (red dashed line), $|3\rangle$ (yellow dotted line) and $|4\rangle$ (green solid line) as functions of time in the double-excited model described by $H_{I}$ in Eq.(3). The horizontal axe is in units of ns.

so that the vector $\left|D_{2}\right\rangle$ initial at north point undergoes an adiabatic evolution along a closed path with a solid angle $\gamma_{c}=\pi / 2$ in the BS2, where

$$
\eta=\frac{\pi}{2}[1+\tanh (\alpha t)]
$$

Parameter $\alpha$ characterizes the bandwidth of pulses. Having the designed pulses (12), now we can test our main theoretical results (10) with the aid of numerical calculations. In order to do the calculation, we first assume that the system is initially populated in state $|1\rangle$. Then we numerical solve the time-dependent Schrödinger equation described by Hamiltonian in Eq. (3) with $\omega_{0}=20 \mathrm{MHz}$, $\alpha=1 \mathrm{MHz}$. In Fig. 3, we plot the norms of amplitudes for all four states as functions of time. It can be found that there are no population in the two excited states (yellow dotted and green solid lines) before and after applying the pulses, however the amplitude of the initial state $|1\rangle$ (blue dot-dashed line) has changed from 1 to $1 / \sqrt{2}$, and the amplitude of the degenerate state $|2\rangle$ increases from 0 to $1 / \sqrt{2}$. Therefore, a rotation of the qubit about $x$ with an angle $\pi / 2$ has been obtained. The non-vanishing amplitude of the double excited states during the adiabatic evolution depicts the non-adiabatic transition between two dark states.

Notice that the optical transitions in NV centers are original associated to an orbital-doublet, spin-triplet excited state and the spin-triplet ground state, the actual operation $U$ is presented by $9 \times 9$ matrices. To check how well our theory works for actualy nine-level systems, we use the operator fidelity [29] $F=\left|\operatorname{Tr} U_{C}^{\dagger} U\right| / 2$ as a measure of how accurate the ideal gate $U_{C}$ can be achieved. Taking the spin-orbit and spin-spin parametersf from Ref.[14], and letting the Rabi frequency amplitudes, the pulse widths and the detuning are same as those in the above, we numerical solve the equation for the unitary evolution operator with the initial condition $U\left(t_{0}, t_{0}\right)=I$. The actual operation $U$ is obtained at the time $t_{f}$ that the applied pulses have turned off. Then we obtain the operator fidelity on the order of $99.9 \%$. 


\section{CONCLUSION}

In conclusion, we have shown that it is possible to establish an excited-double four-level system in the spintriplet gound state and an orbital-doublet, spin-triplet excited state of the NV center due to the spin-orbit and spin-spin interactions, which allow us to apply two pump and two Stokes laser pulses. It is found that two dark states exist when both the pump and stokes fields maintain two-photon resonance and the intensities of two pump (Stokes) fields take the same values. Consequently, we propose a rotation procedure by encoding the qubit in the ground degenerate states. We first map the information of the qubit on one dark state and its corresponding bright state defined by the pump and Stokes pulses. Then we adiabatically tune the Rabi frequencies and phases of the pump and Stokes pulses by keeping nonvanishing two-photon resonance so that the NV center undergoes a cyclic evolution. The adiabatical process first transfers some components of the bright state to the double excited states, afterwords, bring these components back to the bright state accompanied by a phase shift $\gamma_{c}$. Therefore, a rotation of the qubit is acheived due to the non-Abelian geometric phase produced by the nonadiabtic transition between the two degenerate dark states. The axis and angle of rotation is determined by the parameters of the laser fields.

This work is supported by the Program for New Century Excellent Talents in University (NCET-080682), NSFC No. 11074071, and No. 11105050, NFRPC 2012CB922103, PCSIRT No. IRT0964, the Key Project of Chinese Ministry of Education (No. 210150), the Research Fund for the Doctoral Program of Higher Education No. 20104306120003, Hunan Provincial Natural Science Foundation of China(11JJ7001), and Scientific Research Fund of Hunan Provincial Education Department (No. 11B076). We thanks Prof. Renbao Liu and Dr. Nan Zhao for useful discussions.
[1] R. Bhandari, Phys. Rep. 281, 1 (1997).

[2] M. V. Berry, Proc. R. Soc. London, Ser. A 392, 45 (1984).

[3] F. Wilczek and A. Zee, Phys. Rev. Lett. 52, 2111 (1984).

[4] J. Dalibard, F. Gerbier, G. Juzeliūnas, P. Öberg, Rev. Mod. Phys. 83, 1523 (2011).

[5] Chang-Pu Sun, Mo-Lin Ge, Phys. Rev. D 41, 1349 (1990).

[6] A.H. Castro Neto, F. Guinea, N.M.R. Peres, K.S. Novoselov, and A.K. Geim, Rev. Mod. Phys. 81, 109 (2009).

[7] P. Zanardi and M. Rasetti, Phys. Lett. A 264, 94 (1999); J. Pachos, P. Zanardi and M. Rasetti, Phys. Rev. A 61, 010305 (1999).

[8] J. Wrachtrup, and F. Jelezko, J. Phys.: Condens. Matter 18, S807 (2006).

[9] M.V.G. Dutt, L. Childress, L. Jiang et al. Science 316, 1312 (2007).

[10] A.D Greentree, P. Olivero, M. Draganski et al. J. Phys.: Condens. Matter 18, S825 (2006).

[11] A.M. Stoneham, A.H Harker and G.W Morley, J. Phys.: Condens. Matter 21, 364222 (2009).

[12] J. R. Weber, W. F. Koehl, J. B. Varley et al. PNAS 107, 8513 (2010).

[13] A. Lenef and S. C. Rand, Phys. Rev. B 53, 13441 (1996).

[14] M W Doherty, N B Manson, P Delaney and L C L Hollenberg, New J. Phys. 13, 025019 (2011).

[15] J R Maze, A Gali, E Togan, et al. New J. Phys. 13, 025025 (2011).
[16] F. Jelezko, T. Gaebel, I. Popa, A. Gruber, J. Wrachtrup, Phys. Rev. Lett. 92, 076401 (2004).

[17] P. Neumann, R. Kolesov, B. Naydenov et al. Nat. Phys. 6, 249 (2010).

[18] Fazhan Shi, Xing Rong, Nanyang Xu et al. Phys. Rev. Lett. 105, 040504 (2010).

[19] G. D. Fuchs, G. Burkard, P.V. Klimov and D.D. Awschalom, Nat. Phys. 7, 789 (2011).

[20] R. Hanson, V. V. Dobrovitski, A. E. Feiguin, O. Gywat, D. D. Awschalom, Science 320, 352 (2008);

[21] Nan Zhao, Jian-Liang Hu, Sai-Wah Ho, Tsz-Kai Wen, R. B. Liu, Nat. Nanotech. 6, 242 (2011).

[22] Nan Zhao, Zhen-Yu Wang, Ren-Bao Liu, Phys. Rev. Lett. 106, 217205 (2011).

[23] K. Dholakia and T. Čižmár, Nat. Photon. 5, 335 (2011).

[24] D.V. Thourhout and J. Roels, Nat. Photon. 4, 211 (2010).

[25] C. Kurtsiefer, S. Mayer, P. Zarda, and H. Weinfurter, Phys. Rev. Lett. 85, 290 (2000).

[26] A. Batalov, C. Zierl, T. Gaebe et al. Phys. Rev. Lett. 100, 077401 (2008).

[27] C. Santori, P. Tamarat, P. Neumann et al. Phys. Rev. Lett. 97, 247401 (2006).

[28] E. Togan, Y. Chu, A. S. Trifonov et al. Nature 466, 730 (2010).

[29] Xiaoguang Wang, Zhe Sun, and Z. D. Wang, Phys. Rev. A 79, 012105 (2009). 